\title{
LncRNA MBNL1-AS1 Represses Proliferation and Cancer Stem-Like Properties of Breast Cancer Through MBNL1-AS1/ZFP36/CENPA Axis
}

\section{Yu Ding}

The Second Affiliated Hospital of Harbin Medical University

\section{Yingjie Li}

The Second Affiliated Hospital of Harbin Medical University

\section{Yunqiang Duan}

The Second Affiliated Hospital of Harbin Medical University

\section{Wan Wang}

China-Japan Union Hospital of Jilin University

\section{Wei Zheng}

The Second Affiliated Hospital of Harbin Medical University

\section{Weilun Cheng}

The Second Affiliated Hospital of Harbin Medical University

\section{Yuan Qi}

The Second Affiliated Hospital of Harbin Medical University

\section{Jianyuan Feng}

The Second Affiliated Hospital of Harbin Medical University

\section{Ziang Chen}

The Second Affiliated Hospital of Harbin Medical University

\section{Tianshui Yu}

The Second Affiliated Hospital of Harbin Medical University

\section{Anbang Hu}

The Second Affiliated Hospital of Harbin Medical University

\section{Fei Ma}

The Second Affiliated Hospital of Harbin Medical University

\section{Baoliang Guo ( $\sim$ baoliangguo2020@hrbmu.edu.cn )}

The Second Affiliated Hospital of Harbin Medical University

\section{Research Article}

Keywords: LncRNA MBNL1-AS1, breast cancer, ZFP36, CENPA, proliferation, stemness 
Posted Date: December 30th, 2021

DOI: https://doi.org/10.21203/rs.3.rs-1206043/v1

License: (c) (i) This work is licensed under a Creative Commons Attribution 4.0 International License. Read Full License 


\section{Abstract}

Background: Emerging studies suggested the notion that long noncoding RNAs (IncRNAs) were key regulators of cancer progression. In this research, the expression and roles of MBNL1-AS1 were explored in breast cancer $(\mathrm{BC})$.

Methods: In the present research, the MBNL1-AS1 expression in breast cancer tissue, as well as in cell line was studied by qRT-PCR assays. The effects of MBNL1-AS1 on proliferation and stemness were evaluated by MTT assays, colony formation assays, orthotopic breast tumor mice models, and sphere formation assays. Flexmap 3D assays were performed to show that MBNL1-AS1 downregulated the Centromere protein A (CENPA) secretion in BC cells. Western blot, RNA pull-down assays, RNA immunoprecipitation (RIP) assays, and Fluorescence in situ hybridization (FISH) were conducted to detect the mechanism.

Results: The results revealed that the expression levels of MBNL1-AS1 were downregulated in breast cancer tissues and cell lines. In vitro and in vivo studies demonstrated that overexpression of MBNL1-AS1 markedly inhibited BC cells proliferation and stemness. RNA pull-down assay, RIP assay, western blot assay, and qRT-PCR assay showed that MBNL1-AS1 downregulated CENPA mRNA via directly interacting with Zinc Finger Protein 36 (ZFP36) and subsequently decreased the stability of CENPA mRNA. Restoration assays also confirmed that MBNL1-AS1 suppressed the CENPA-mediated proliferation and stemness in breast cancer cells.

Conclusions: We elucidated a new mechanism for how MBNL1-AS1 regulated the phenotype of BC and targeting the MBNL1-AS1/ZFP36/CENPA axis might serve as therapeutic targets for BC patients.

\section{Introduction}

Female breast cancer has surpassed lung cancer as the most common cancer, with about 2.3 million new cases $(11.7 \%)(1)$. It is the main cause of cancer-associated death for women around the world(2).Approximately $15.4 \%$ of the women died of breast cancer, although the diagnosis and treatment strategies improved greatly over the past several decades(1). The abnormal proliferation of tumor cells is a striking feature of malignant tumors. Cancer stem cells (CSCs) have vital roles in intra- and intertumoral heterogeneity, which are related to tumor progression, treatment resistance, and disease relapse(3, 4).Therefore, exploring the potential molecular mechanisms underlying breast cancer proliferation and stemness is of paramount importance in identifying effective and novel therapeutic strategies.

LncRNAs, classes of non-coding RNAs (ncRNAs) have greater than 200 nucleotides length $(5,6)$. LncRNAswere able to modulateseveral phenotypes such as proliferation, metastasis, stemness and progression of cell cycle(7-10). Accumulating research hadshowed that the expression pattern of IncRNAswas correlated with cancer progression,proliferation, and stemness(10-13)[9-11].Emerging evidence demonstrated that IncRNAs regulated the expression level of a target gene by binding to RNAbinding proteins(RBPs).For instance,the LncRNAs interacted with the RBPs and subsequently regulated 
cancers progression $(14,15)$. These researchersshowed that IncRNAswere pivotal players in cancer pathogenesis and vital new biomarkers in cancer early detection and therapy. Recently,two cohorts were analyzed to find LncRNAs of differential expression by research in BC. MBNL1-AS1 was regarded as one of the down-regulated IncRNAs in BC(13). However, the phenotype and mechanism of MBNL1-AS1 in BC have not been detected.

CENPA (a $17 \mathrm{kDa}$ variant of histone $\mathrm{H} 3$ )was located in the active centromeres(16, 17). A previous study has found that CENPAwas correlated with human pluripotent stem cell self-renewal(18). Low expression of CENPA induced cell cycle arrest and promoted apoptosis(19). Subsequent evidence showed that upregulation of CENPA promoted initiation and progression in several cancers(17, 20). Higher expression of CENPAwas correlated with increased invasiveness and higher-grade cancers $(19,21)$. In contrast, downregulation of CENPAshowed to inhibit the HCC cells proliferation(19). What's more, CENPA had predictive value in breast cancer and could contribute to disease progression as a marker of proliferation $(21,22)$. In thisresearch, we found thatMBNL1-AS1 markedlydecreased the expression of CENPAMRNA.

Here, we showed that MBNL1-AS1 in BC was down-regulated and the decreased expression of MBNL1AS1 was correlated with survival. MBNL1-AS1 inhibitedBC proliferation and stem-cell properties were confirmed by functional studiesin vitro and in vivo. Mechanistically, MBNL1-AS1 directly interactedwith ZFP36, an RNA-binding protein, subsequently reduced the stabilization of CENPA mRNA. This study might bring new insights into therapeutic targets for BC.

\section{Methods}

\section{Cell lines and human $B C$ specimens}

Human BC cell lines (MCF-7, MDA-MB-468 and MDA-MB-231) and the immortalized normal breast cell line MCF-10A were purchased from the Cell Bank of Chinese Academy of Sciences (Shanghai, China). Indicated cells were cultured in DMEM containing 10\% FBS (Thermo Fisher Scientific) was supplied at $37^{\circ} \mathrm{C}$ in a humidified atmosphere of 5\% CO2. $60 \mathrm{BC}$ samples were obtained from the Department of General Surgery, the Second Affiliated Hospital of Harbin Medical University. Fresh BC tissues and paired adjacent normal tissues were frozen in liquid nitrogen immediately after surgical excision and saved at $-80^{\circ} \mathrm{C}$. This research was approved by the Ethic Committee of Harbin Medical University.

\section{qRT-PCR assay}

To isolate total-RNA, the TRIzol reagent (Life Technologies, Carlsbad, CA, USA) was performed. For cDNA synthesis, the total RNA was retrotranscribed with the PrimeScript® RT Reagent Kit (Takara). The SYBR Premix Ex Taq ${ }^{\text {TM }}$ Kit (Takara, Tokyo, Japan) was utilized to carry out qPCR. And RT-PCR was conducted on a 7500 RealTime PCR System. GAPDH was measured as the internal control. The sequences of the primers that were used as follows: for MBNL1-AS1: 5'- CTCCCGCTTCTTCTACCGAC -3' (forward), 5'TTGGTGCATTTTAAGGCGGC -3' (reverse); for CENPA: 5'- GATTCTGCGATGCTGTCTG -3' (forward), 5'- 
GCCTTTGGAACGGTGTT -3'(reverse); for ZFP36: 5'- TCCACAACCCTAGCGAAGAC -3' (forward), 5'GAGAAGGCAGAGGGTGACAG -3' (reverse); for GAPDH, 5'- CTCCTCCACCTTTGACGCTG -3' (forward), 5'TCCTCTTGTGCTCTTGCTGG - 3' (reverse). All data were calculated by $2^{-\triangle \triangle C T}$ method.

\section{Cell transfection}

In order to assess the MBNL1-AS1 overexpression vector, the lentiviral vector-MBNL1-AS1 and lentiviral vector -convirus (Genepharma, Shanghai, China) were transfected in cells. sh-MBNL1-AS1\#1,sh-MBNL1AS1\#2 and sh-control were obtained from GenScript (Nanjing, China). The qRT-PCR assays were used to detect the expression of MBNL1-AS1. The sequences of the MBNL1-AS1 targeting shRNAs were: for MBNL1-AS1-shRNA\#1: 5'-

GATCCGAACGAAAGGAGCAGGGTATTTCAAGAGAATACCCTGCTCCTTTCGTTTTTTTA-3' (sense), 5'AGCTTAAAAAAACGAAAGGAGCAGGGTATTCTCTTGAAATACCCTGCTCCTTTCGTTCG-3' (anti-sense); for MBNL1-AS1-shRNA\#2: 5'-

GATCCGCCAGAACCTAGTCTCATGTTTCAAGAGAACATGAGACTAGGTTCTGGTTTTTA-3' (sense), 5'AGCTTAAAAACCAGAACCTAGTCTCATGTTCTCTTGAAACATGAGACTAGGTTCTGGCG-3' (anti-sense); for NC-shRNA: 5'- GATCCCCTTCTCCGAACGTGTCACGTTTCAAGAGAACGTGACACGTTCGGAGAATTTTT -3' (sense), 5'- AGCTAAAAATTCTCCGAACGTGTCACGTTCTCTTGAAACGTGACACGTTCGGAGAAGGG -3' (antisense). siRNA, si-ZFP36, si-NC were obtained from RiboBio (Shanghai, China). Cells were transfected using the Lipofectamine 2000 (Invitrogen).

\section{MTT study}

Transfected cells were seeded in a 96-well plate whichhad $3 \times 10^{3}$ cells/well. Each well was added with $10 \mu \mathrm{L}$ MTT $(5 \mathrm{mg} / \mathrm{mL})$ for different periods of time. After 4 hours of incubation, $150 \mu$ of dimethyl sulfoxide was added to dissolve the precipitates. The absorbance was then measured by detection at 560 $\mathrm{nm}$ using a microplate reader. The experiment was performed independently in triplicate.

\section{Colony formation analysis}

The BC cells were harvested and plated in a 6 -well plate $\left(0.2 \times 10^{3}\right.$ cells/well) and cultured in incubators. After 14 days of culture, the plate was washed 3 times with PBS. The indicated BC cells were dried at $37^{\circ} \mathrm{C}$ for 15 minutes. Then the cell colonies formed was numbered under a digital camera.

\section{Sphere formation assay}

A total of $5 \times 10^{3}$ cells were plated in 6-well plates (Corning Life Science). EGF ( $20 \mathrm{ng} / \mathrm{ml}$, Invitrogen), bFGF (10 ng/ml, Invitrogen), and 2\% B27 (Invitrogen, Carlsbad, CA) was formulated in a medium. After 2 weeks, the number of spheroids was counted manually by a microscope.

\section{Flow cytometry assay}


Using flow cytometry with the use of propidium iodide (Sigma-Aldrich, MO China) staining $(5 \mu \mathrm{g} / \mathrm{ml}$ ), cell cycle analysis was performed after transfection. Then, the indicated cells were added with chilled ethanol and saved at $4{ }^{\circ} \mathrm{C}$ overnight. Then, the BC cells were centrifuged and resuspended in PBS and appended with $100 \mu \mathrm{L}$ RNase at room temperature. After $30 \mathrm{~min}, 100 \mu \mathrm{l} \mathrm{PI}$ was appended, cells were incubated at $37^{\circ} \mathrm{C}$ in dark for 60 minutes. Cell cycle was determined by FACScaliber Flow Cytometer.

\section{Western blot}

Total protein from tumor lysateby a lysis buffer (Beyotime, Jiangsu, China) and separated using 10\% SDS-PAGE. A PVDF membrane was transferred by the indicated protein, which was blocked with $5 \%$ nonfat dry milk. Primary antibodies, namely, Ab against ZFP36, Ab against CENPA, and Ab against GAPDH was incubated with the membrane. Subsequently, the secondary antibodies were incubated with the membrane for $1 \mathrm{~h}$. After developed by enhanced chemiluminescent and exposure, immunoreactive protein band intensities were analyzed by Image software.

\section{RNA pull-down}

The biotin-labeled MBNL1-AS1 plasmid and biotin-labeled antisense RNA plasmid were respectively transfected in BC cells. Biotin-labeled RNA was bound to the Streptavidin agarose Beads, next mixed with indicated cell lysates. Indicated bound RNAs were isolated from the beads by washed and boiled for normal western blot. The assays were experimented in RNase-free conditions.

\section{RNA immunoprecipitation (RIP) assay}

RIP was performed with the use of the Magna RIP kit (Millipore, USA). The spectrophotometer (Thermo Scientific, USA) was used to examine the RNA concentration, and the bio-analyzer (Agilent, USA) was utilized to detect the RNA quality. The input control was the total RNA. Following, qRT-PCR analysis was used to test the results in order to demonstrate the bound targets.

\section{Magnetic Luminex® performance assay}

Magnetic Luminex® performance assay was performed based on our previous study(14). Briefly, NC or MBNL1-AS1 were transfected in MDA-MB-231 cells, which were incubated for 1 day. Following centrifugation, the supernatant was collected. Then the following human cytokines: PLK1, PAF, CENPA, YB-1, TWIST, YY1, KLF4, CUG2, E2F8, SALL4, RAE1 and PTPA were analyzed by FlexMAP 3D (Luminex®) platform.

\section{Fluorescence in situ hybridization (FISH) assay}

The MBNL1-AS1 subcellular localization was assessed by a FISH kit was obtained from Guangzhou RiboBio Co., Ltd. The 4\% paraformaldehyde-fixed cells were fixed in PBS. After washed by phosphate buffered solution with tween for three times, the anti-fluorescence quencher sealed the indicated cells, the images were taken by the FV1000 laser microscope (Olympus, Japan). 


\section{mRNA decay assay}

Stable cells were added with $5 \mu \mathrm{g} / \mathrm{mL}$ actinomycin D. Following, qRT-PCR was performed to determine the CENPA mRNA. The data was performed independently in triplicate.

\section{Animal studies}

Animal studies were ratified by ethics committee of Harbin Medical University (Harbin, China). The BC cells were injected in the flank of four-week-old female nude mice (each group has 3 mice). The volume of tumor was recorded every week. Euthanasia of mice by overdose of pentobarbital sodium $(200 \mathrm{mg} / \mathrm{kg}$, intraperitoneally) and tumors were measured after 4 weeks.

\section{Statistical analysis}

All analysis were experimented three times at least. The measurement data in this study were exhibited as means \pm SD. Student's t-test was applied to compare the numeric variables between two groups. $R$ software package version 3.0.0 and Graphpad Prism 5 were used. A p-value $<0.05$ was regarded as significant, P-value $<0.01$ was very significant.

\section{Results}

\section{MBNL1-AS1 expression was downregulated in BC cells which was correlated with poor prognosis}

MBNL1-AS1 was regarded as one of the LncRNA suppressor genes associated with breast cancer, according to the bioinformatics analysis(13). However, expression and function in breast tissue have not been confirmed yet.Therefore, the MBNL1-AS1 expression was detected inthe cancer genome atlas (TCGA) database. As indicated in Figure1A, the MBNL1-AS1expression pattern was marked lower than normal tissues in BC tissues. The MBNL1-AS1 levels in different BC subtypes were explored for the following study. The MBNL1-AS1 levels in tumor tissues were found markedly decreased in HER2+, Luminal A, and Luminal B subtypes compared with normal tissues. However, no statistical significance of MBNL1-AS1 expression was found in basal-like subtype between tumor tissues and normal tissues (Figure 1B). Next, we examined the MBNL1-AS1 expression level in 60 BC tissues and normal breast tissues. According to using the qRT-PCR,MBNL1-AS1 expression significantly decreased in the BC tissues and the normal tissues (Figure 1C). Then, we linked the expression of MBNL1-AS1 with the clinicopathological features of the BC patients. We observed that the expression of MBNL1-AS1 had negatively relationship with TNM stage and lymph node metastasis. (Figure 1D and Table 1). Then, we detected the MBNL1-AS1 expression in severalBC cell lines. The MBNL1-AS1 expression levelswere observed to be much lower in BC cell lines, particularly in MDA-MB-468 and MDA-MB-231 cell lines withthe higher metastatic features, compared with the immortal MCF-10A cells and low-metastatic BC cell line-MCF-7 (Figure 1E). Given this expression mode, we selected MCF-7 and MDA-MB-231 cell lines to perform the following functional study. We used a Kaplan-Meier Plotter to make clear the relationship between the expression levels of MBNL1-AS1 and the patients' survival. The results showed that 
lowerMBNL1-AS1 expression was markedly correlated to worse relapse-free survival(RFS) (Figure 1F). Altogether, the resultsdemonstrated that the downregulated MBNL1-AS1 in BC might associate with a poor prognosis.

\section{MBNL1-AS1 inhibited proliferation and stemness of BCin vitro and in vivo}

We examined the biological functions of MBNL1-AS1 in BC by observing the relationship between expression of MBNL1-AS1 and BC prognosis.MBNL1-AS1 levels weresilenced by shRNA in MCF-7 cellsand overexpressed in MDA-MB-231 cells.RT-PCR analysis wasalso utilized to confirm the efficiency (Figure2A). As shown in Figure2B, knockdown of MBNL1-AS1 by shRNA markedly enhanced the growth of MCF-7 cells. In contrast, MBNL1-AS1 over-expressionsignificantly inhibited the proliferation abilities of MDA-MB-231 cells. Colony formation assays further confirmed the antiproliferation of MBNL1-AS1 in the BC cells (Figure2C). Next,the sphere formation assay was usedto detectthe stemness of BCcells. The stemness propertiesof MCF-7 cells increased significantly by knockdown of MBNL1-AS1, whereas the stemness properties of MDA-MB-231 cells decreased markedly as overexpression of MBNL1-AS1 (Figure2D).Flow cytometry assays confirmed that MBNL1-AS1 influencedthe cell cycle, evidenced by the G0/G1- cells were increased and the S- and G2/M- cells were reduced (Figure2E). To confirm the effect of MBNL1-AS1 in vivo, MCF-7 cells transfected with sh-MBNL1AS1 showed dramatically increased tumorigenic abilities in vivo. Tumorigenic abilitieswere significantly inhibited in MBNL1-AS1 over-expressionmicecompared with control mice(Figure2F). Collectively, the results revealed that MBNL1-AS1 suppressed the BC cells proliferation and stemnessabilities.

\section{MBNL1-AS1 suppressed the expression of CENPAby reducing the stability of CENPA mRNA}

To elucidate the mechanism which MBNL1-AS1 suppress the proliferation and stemness of breast cancer cells, we analyzed some known factors closely related to the proliferation and stemness of BCbyflexmapliquichip assays.The results showed that overexpression of MBNL1-AS1 in MDA-MB-231 cells significantly inhibited the CENPAsecretion, however, other proteinsdid not have statistical significance (Figure3A, B). We, therefore, set out to detect the regulatory effect of MBNL1-AS1 on CENPAin BCcells. Interestingly, MBNL1-AS1 significantly reduced the levels of CENPAprotein and mRNA in $B C$ cell lines (Figure3C, D). Since the subcellular location of LncRNAs determined the functions, FISH assaywas utilizedto detect MBNL1-AS1 and CENPA immunofluorescence staining to explore the possible mechanism of MBNL1-AS1 downregulating CENPA expression (Figure3E). We detected that MBNL1-AS1 localized mainly in cytoplasm of breast cancer cells, implying that CENPAmight be regulated in a posttranscriptional manner. As shown in Figure3E, MBNL1-AS1 knockdown markedly upregulated the CENPAexpression in MCF-7 cells and vice versa in another cell lines. We next detectedMBNL1AS1 whetheror notregulated the CENPAmRNA in breast cancer cells. As expected,after the treatmentof actinomycin D (an RNA synthesis inhibition agent), the CENPA mRNA half-life in MBNL1-AS1-silenced MCF-7 cells wasdramaticallyprolonged while the CENPA mRNA half-life shortened after MBNL1-AS1 was transferred to MDA-MB-231 cells(Figure3F). In conclusion, MBNL1-AS1 inhibited the CEPNA expression by reducing the stability of CENPA mRNA. 


\section{MBNL1-AS1 directly interacted with ZFP36 and subsequently reduced the stability of CENPA mRNA}

To investigate how MBNL1-AS1 regulated CENPA, the RBPDB software was used to predicted target proteins of MBNL1-AS1. ZFP36 was found that could directly bind with MBNL1-AS1.Besides, researchers showed that ZFP36 can specifically bind to AU-rich elements (ARE) in the mRNA 3'UTR and subsequently induce mRNA decay $(23,24)$. RIP assays were used to examine whether MBNL1-AS1 was physically associated with ZFP36. As indicated in Figure4A, these results demonstrated that MBNL1-AS1 could directly bind with ZFP36 in indicated cells. RNA pull-down analysisfurther demonstrated the interaction of MBNL1-AS1 and ZFP36. ZFP36 proteins were pulled down by MBNL1-AS1, but the antisense RNA was not pulled down (Figure4B). To examine whether MBNL1-AS1 bound to ZFP36 and modulated the CENPA mRNA stability, we tested the CENPA mRNA half-life in indicated cells after ZFP36 was silenced. Before that, western blot assay was used toexamine the ZFP36 knockdown efficiency in the BC cells (Figure4C). As shown in Figure4C, D, MBNL1-AS1 could not affect the CENPA mRNA stability after ZFP36 silenced, nor decrease the protein pattern of CENPAin indicated cells. To conclude, MBNL1-AS1 directly interacted with ZFP36 and reduced the CENPA mRNA stability.

\section{MBNL1-AS1 suppressed proliferation and stemness of breast cancer cells by interacting with ZFP36}

To further validate our findings, MTT assays, colony formation assays, and sphere formation assays were performed to examine the function of ZFP36 in breast cancer cell lines and xenograft tumor models. ZFP36 knockdown significantly enhanced the growth of MCF-7 cells, however knockdown of MBNL1AS1did not revert the proliferation abilities (Figure5A, B). The same results also showed that MBNL1-AS1 overexpression did not affect the growth of MDA-MB-231 cells after ZFP36 knockdown. Next,we examined the stemness abilities of indicated cells. As indicated in Figure5C,ZFP36 knockdown enhanced the stemness abilities of MCF-7 cells. However, knockdown of MBNL1-AS1 did not revert the stemness abilities of indicated cells.Similarly, sphere formation assays showed that MBNL1-AS1 knockdown or overexpression failed to revert the increase of sphere numbers in si-ZFP36 transfected BC cells.Meanwhile, the results in vivo assays also confirmed that transfection of MBNL1-AS1 or si-MBNL1AS1 did not revert the tumorigenic abilities after ZFP36 knockdown in orthotopic breast cancer mice models(Figure5D). Ourdata revealed that the interaction of MBNL1-AS1 and ZFP36 inhibitedthe proliferation and stemness of BC cells.

\section{Discussion}

LncRNAsare known as diagnostic markers forkinds of cancers including BC. Previous research demonstrated various IncRNAs as vital players in $B C$ progression $(13,25)$. In the presentresearch, the MBNL1-AS1 expression in breast cancer patients and its functions in BCcells were detected and investigated. The results indicated that expression of MBNL1-AS1 markedly decreased in breast cancer and high-metastatic BC cell lines, which wasin line with the studies revealed that MBNL1-AS1 levels were down-regulated incolorectal cancer,NSCLC, and bladder cancer.(26-30). Thesedemonstrated that MBNL1AS1 mightbe a reliable biological marker to diagnose BC.The clinical significance of MBNL1-AS1 still 
needs to be further verified in moresamples for the limitations of the present study. Moreover, MBNL1-AS1 inhibited the proliferation and stemness of breast cancercells in vitro and inhibited the tumorigenesis of breast cancer cells in vivo was demonstrated by these results of gain- or loss of- function studies. These results indicated that MBNL1-AS1 played antioncogenic roles in BC.

Mounting evidence showed that IncRNAs regulated the downstream genes expression by binding to RBPs and competing endogenous RNAs (ceRNAs) $(14,25,31,32)$.ZFP36was known as RBP, which bound to the target mRNAs untranslated regionssubsequently reducedtheir stability (31, 33-35).Itwas studiedthat the ZFP36 expression was downregulated in BC and regulated the stability of CENPA mRNA(34). However, the upstream regulating factors except ZFP36 are still being researched.Our study demonstrated that the ability of ZFP36 to modulatethe stability of CENPA mRNA was regulated by MBNL1-AS1. MBNL1-AS1 directly interacted with ZFP36 and subsequently reduced the stabilization of CENPA mRNA. Similar regulatorymechanisms were also found in previous studies $(31,36)$.In this research, the stability of CENPAmRNA was modulated by MBNL1-AS1. However, the possibility of translation of the MBNL1-AS1 control CENPA mRNA has not yet been detected. Thus, to contribute to the development of howMBNL1AS1 regulated the CENPAexpression, further study is still needed.

MBNL1-AS1 was regarded as one of the tumor suppressorsIncRNAs in BC(13). Nevertheless, no studies had confirmed the MBNL1-AS1 expressionpattern in BC. Therefore, the expression pattern of MBNL1-AS1 was detected for the first time in breast cancer tissues and cell lines. MBNL1-AS1 was confirmed that the expression in breast cancerand highly metastatic cells was downregulated. That result was consistent with the bioinformatic data from TCGA. In functional assays, ananti-stemness and anti-proliferation function of MBNL1-AS1 was showed by decreasing CENPA expression. In accordance with our study, theresearchersalso confirmed that MBNL1-AS1 was a tumor-suppressive IncRNA (26-29). We demonstrated that MBNL1-AS1 attenuatedthe abilities of breast cancer stemness and proliferation through reduced the stability of CENPA mRNA, which unraveled a novel mechanism of MBNL1-AS1. Undoubtedly, the MBNL1-AS1 and the interplay network and the vital roles of MBNL1-AS1 in BC were enriched and verified by this study.

\section{Conclusions}

We demonstrated that MBNL1-AS1 levels were downregulated in BC tissues, which were correlated with prognosis. In vitro and in vivo assays unraveledtheanti-stemness and anti-proliferation roles of MBNL1AS1. In mechanism, MBNL1-AS1 interacted with ZFP36 and subsequently reduced the stabilization of CENPA mRNA(Figure5E). Therefore, we elucidated a novel mechanism for how MBNL1-AS1 regulated the phenotype of BC and targeting the MBNL1-AS1/ZFP36/CENPAaxis might serve as therapeutic targets for breast cancer patients.

\section{Declarations}

\section{Data availability statement}


The data used or analyzed during the current study are available from the corresponding author on reasonable request.

\section{Conflicts of interest}

None

\section{Funding}

The work was founded by grants from the National Natural Science Foundation of China (8187101021, H1622) and the National Natural Science Foundation of China (8200102587, H1622).

\section{Acknowledgements}

The work was founded by grants from the National Natural Science Foundation of China (8187101021, H1622) and the National Natural Science Foundation of China (8200102587, H1622).

\section{Ethics approval and consent to participate}

This study was approved by the Ethical Committee of Harbin Medical University. The study was performed according to the ethical standards of Declaration of Helsinki and patient informed consent for the use of tissues was obtained prior to the initiation of the study. Additionally, the animal experiments were performed in accordance with the Guide for the Administration of Affairs Concerning Experimental Animals. The study was carried out in compliance with the ARRIVE guidelines.

\section{Consent to publish}

Not applicable.

\section{Authors' contribution}

Yu Ding,Yunqiang Duan and Fei Ma conceivedthe project; Yingjie Li and Wan Wang acquired and finished analysis of the data; Wei Zheng, Weilun Cheng, Yuan Qi,Jianyuan Feng, Ziang Chen,Tianshui Yuand Anbang Huperformed the experiments; Baoliang Guo, Fei Ma and Yu Ding wrote the manuscript.

\section{References}

1. Sung H, Ferlay J, Siegel RL, Laversanne M, Soerjomataram I, Jemal A, et al. Global cancer statistics 2020: GLOBOCAN estimates of incidence and mortality worldwide for 36 cancers in 185 countries. CA: a cancer journal for clinicians. 2021.

2. Siegel RL, Miller KD, Fuchs HE, Jemal AJCACJfC. Cancer Statistics, 2021. 2021;71.

3. Brooks MD, Burness ML, Wicha MSJCSC. Therapeutic Implications of Cellular Heterogeneity and Plasticity in Breast Cancer. 2015;17(3):260-71. 
4. Visvader JE, Lindeman GJJCSC. Cancer stem cells: current status and evolving complexities. 2012;10(6):717-28.

5. Cech, T. R, Steitz, J. AJC-CM-. The Noncoding RNA Revolution-Trashing Old Rules to Forge New Ones. 2014.

6. Guttman, M., Russell, P., Ingolia, N. T, et al. Ribosome Profiling Provides Evidence that Large Noncoding RNAs Do Not Encode Proteins. 2013.

7. Bhan A, Soleimani M, Mandal SS. Long Noncoding RNA and Cancer: A New Paradigm. Cancer research. 2017;77(15):3965-81.

8. Mercer TR, Dinger ME, Mattick JS. Long non-coding RNAs: insights into functions. Nature reviews Genetics. 2009;10(3):155-9.

9. Hu WL, Jin L, Xu A, Wang YF, Thorne RF, Zhang XD, et al. GUARDIN is a p53-responsive long noncoding RNA that is essential for genomic stability. Nature cell biology. 2018;20(4):492-502.

10. Tang T, Guo C, Xia T, Zhang R, Jin LJT. LncCCAT1 Promotes Breast Cancer Stem Cell Function through Activating WNT/ $\beta$-catenin Signaling. 2019;9(24):7384-402.

11. Wang G, Chen H, Liu J. The long noncoding RNA LINC01207 promotes proliferation of lung adenocarcinoma. American journal of cancer research. 2015;5(10):3162-73.

12. Yu S, Li N, Huang Z, Chen R, Yi P, Kang R, et al. A novel IncRNA, TCONS_00006195, represses hepatocellular carcinoma progression by inhibiting enzymatic activity of ENO1. Cell death \& disease. 2018;9(12):1184.

13. Xu S, Kong D, Chen Q, Ping Y, Pang D. Oncogenic long noncoding RNA landscape in breast cancer. Molecular cancer. 2017;16(1):129.

14. Zhang Y, Sun J, Qi Y, Wang Y, Ding Y, Wang K, et al. Long non-coding RNA TPT1-AS1 promotes angiogenesis and metastasis of colorectal cancer through TPT1-AS1/NF90/VEGFA signaling pathway. Aging. 2020;12(7):6191-205.

15. Zang X, Gu J, Zhang J, Shi H, Hou S, Xu X, et al. Exosome-transmitted IncRNA UFC1 promotes nonsmall-cell lung cancer progression by EZH2-mediated epigenetic silencing of PTEN expression. Cell death \& disease. 2020;11(4):215.

16. Zeitlin SG. Centromeres: the wild west of the post-genomic age. Epigenetics. 2010;5(1):34-40.

17. Tomonaga T, Matsushita K, Yamaguchi S, Oohashi T, Shimada H, Ochiai T, et al. Overexpression and mistargeting of centromere protein-A in human primary colorectal cancer. Cancer research. 2003;63(13):3511-6.

18. Ambartsumyan G, Gill RK, Perez SD, Conway D, Vincent J, Dalal YJHMG. Centromere protein A dynamics in human pluripotent stem cell self-renewal, differentiation and DNA damage. 2010;19(20):3970-82.

19. Li Y, Zhu Z, Zhang S, Yu D, Yu H, Liu L, et al. ShRNA-targeted centromere protein A inhibits hepatocellular carcinoma growth. PloS one. 2011;6(3):e17794. 
20. Wu Q, Qian YM, Zhao XL, Wang SM, Feng XJ, Chen XF, et al. Expression and prognostic significance of centromere protein $A$ in human lung adenocarcinoma. Lung cancer (Amsterdam, Netherlands). 2012;77(2):407-14.

21. Ma XJ, Salunga R, Tuggle JT, Gaudet J, Enright E, McQuary P, et al. Gene expression profiles of human breast cancer progression. Proceedings of the National Academy of Sciences of the United States of America. 2003;100(10):5974-9.

22. McGovern SL, Qi Y, Pusztai L, Symmans WF, Buchholz TA. Centromere protein-A, an essential centromere protein, is a prognostic marker for relapse in estrogen receptor-positive breast cancer. Breast cancer research : BCR. 2012;14(3):R72.

23. Sanduja S, Blanco FF, Young LE, Kaza V, Dixon DA. The role of tristetraprolin in cancer and inflammation. Frontiers in bioscience (Landmark edition). 2012;17:174-88.

24. Carballo E, Lai WS, Blackshear PJ. Evidence that tristetraprolin is a physiological regulator of granulocyte-macrophage colony-stimulating factor messenger RNA deadenylation and stability. Blood. 2000;95(6):1891-9.

25. Ma F, Liu X, Zhou S, Li W, Liu C, Chadwick M, et al. Long non-coding RNA FGF13-AS1 inhibits glycolysis and stemness properties of breast cancer cells through FGF13-AS1/IGF2BPs/Myc feedback loop. Cancer letters. 2019;450:63-75.

26. Zhu K, Wang Y, Liu L, Li S, Yu W. Long non-coding RNA MBNL1-AS1 regulates proliferation, migration, and invasion of cancer stem cells in colon cancer by interacting with MYL9 via sponging microRNA412-3p. Clinics and research in hepatology and gastroenterology. 2020;44(1):101-14.

27. Cao G, Tan B, Wei S, Shen W, Wang X, Chu Y, et al. Down-regulation of MBNL1-AS1 contributes to tumorigenesis of NSCLC via sponging miR-135a-5p. Biomedicine \& pharmacotherapy = Biomedecine \& pharmacotherapie. 2020;125:109856.

28. Wei $X$, Yang $X$, Wang $B$, Yang $Y$, Fang Z, Yi C, et al. LncRNA MBNL1-AS1 represses cell proliferation and enhances cell apoptosis via targeting miR-135a-5p/PHLPP2/FOXO1 axis in bladder cancer. Cancer medicine. 2020;9(2):724-36.

29. Wei X, Wang B, Wang Q, Yang X, Yang Y, Fang Z, et al. MiR-362-5p, Which Is Regulated by Long NonCoding RNA MBNL1-AS1, Promotes the Cell Proliferation and Tumor Growth of Bladder Cancer by Targeting QKI. Frontiers in pharmacology. 2020;11:164.

30. Li P, Xing W, Xu J, Yuan D, Liang G, Liu B, et al. microRNA-301b-3p downregulation underlies a novel inhibitory role of long non-coding RNA MBNL1-AS1 in non-small cell lung cancer. Stem cell research \& therapy. 2019;10(1):144.

31. Wu JC, Luo SZ, Liu T, Lu LG, Xu MY. linc-SCRG1 accelerates liver fibrosis by decreasing RNA-binding protein tristetraprolin. FASEB journal : official publication of the Federation of American Societies for Experimental Biology. 2019;33(2):2105-15.

32. Hua K, Deng X, Hu J, Ji C, Yu Y, Li J, et al. Long noncoding RNA HOST2, working as a competitive endogenous RNA, promotes STAT3-mediated cell proliferation and migration via decoying of let-7b in triple-negative breast cancer. Journal of experimental \& clinical cancer research : CR. 2020;39(1):58. 
33. Brennan SE, Kuwano Y, Alkharouf N, Blackshear PJ, Gorospe M, Wilson GM. The mRNA-destabilizing protein tristetraprolin is suppressed in many cancers, altering tumorigenic phenotypes and patient prognosis. Cancer research. 2009;69(12):5168-76.

34. Hitti E, Bakheet T, Al-Souhibani N, Moghrabi W, Al-Yahya S, Al-Ghamdi M, et al. Systematic Analysis of AU-Rich Element Expression in Cancer Reveals Common Functional Clusters Regulated by Key RNA-Binding Proteins. Cancer research. 2016;76(14):4068-80.

35. Sanduja S, Kaza V, Dixon DA. The mRNA decay factor tristetraprolin (TTP) induces senescence in human papillomavirus-transformed cervical cancer cells by targeting E6-AP ubiquitin ligase. Aging. 2009;1(9):803-17.

36. Pan QH, Fan YH, Wang YZ, Li DM, Hu CE, Li RX. Long noncoding RNA NNT-AS1 functions as an oncogene in breast cancer via repressing ZFP36 expression. Journal of biological regulators and homeostatic agents. 2020;34(3):795-805.

\section{Tables}

Table 1 Relationship between MBNL1-AS1 expression and clinicopathologic features of BC patients ( $\mathrm{n}=$ 60) 


\begin{tabular}{|c|c|c|c|}
\hline \multirow[t]{2}{*}{ Variable } & \multicolumn{2}{|c|}{ Relative MBNL1-AS1 expression } & \multirow[t]{2}{*}{ P-value } \\
\hline & Low $(n=30)$ & High $(n=30)$ & \\
\hline Age & & & NS \\
\hline$\varangle 50$ & 16 & 13 & \\
\hline$₫ 50$ & 14 & 17 & \\
\hline Histological differentiation & & & NS \\
\hline Well & 9 & 13 & \\
\hline Moderate & 12 & 12 & \\
\hline Poor & 9 & 5 & \\
\hline Tumor size & & & NS \\
\hline$₫ 2 \mathrm{~cm}$ & 8 & 17 & \\
\hline $2-5 \mathrm{~cm}$ & 11 & 6 & \\
\hline$\triangle 5 \mathrm{~cm}$ & 11 & 7 & \\
\hline Lymph node metastasis & & & $\mathrm{P} \otimes 0.05$ \\
\hline Yes & 18 & 9 & \\
\hline No & 12 & 21 & \\
\hline Tumor stage & & & $P \otimes 0.05$ \\
\hline I & 6 & 16 & \\
\hline II & 13 & 9 & \\
\hline III & 11 & 5 & \\
\hline
\end{tabular}

Note: BC patients were divided into MBNL1-AS1 high group and low group according to the analysis of qRT-PCR detection. NS, not significant between different groups. Differences among variables were evaluated by $\chi 2$ or Fisher's exact $\chi 2$-test

Figures 
A

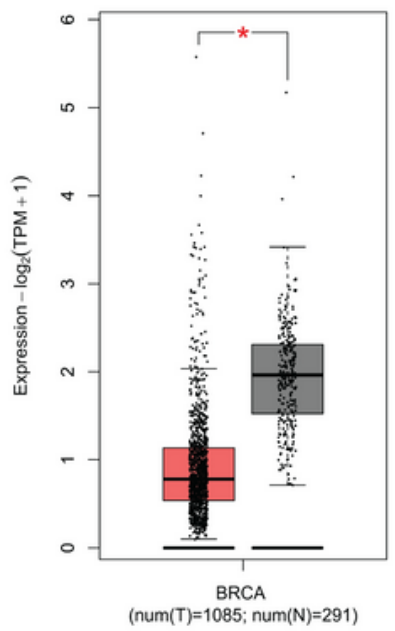

C

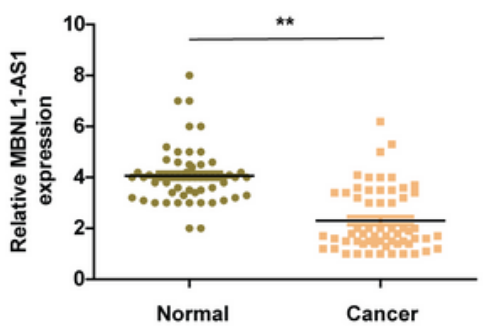

D

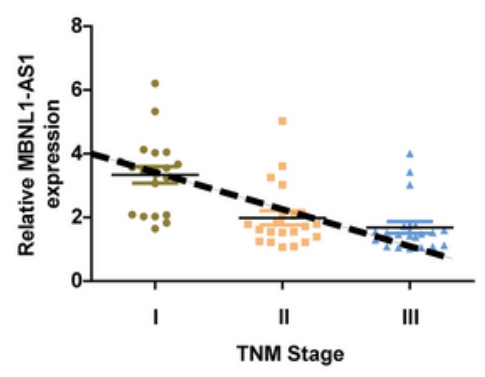

B

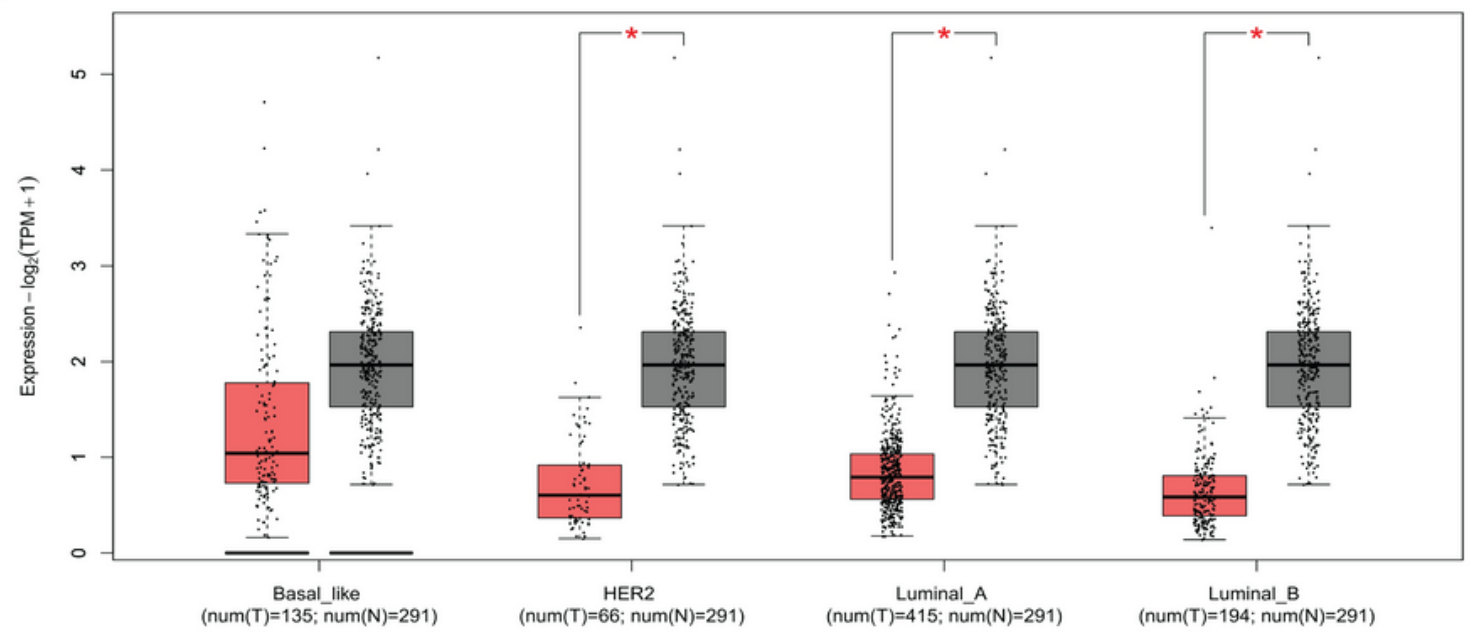

E

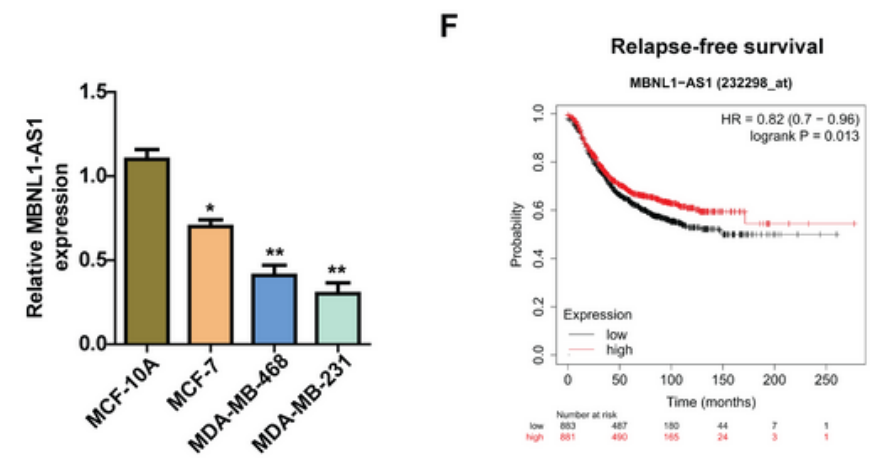

\section{Figure 1}

MBNL1-AS1 was decreased in breast cancer and correlated with survival. (A)The TCGA data set from the GEPIA2 Platform revealed that the expression levels of MBNL1-AS1 were downregulated in BC tissues compared with normal breast tissues.(B)The MBNL1-AS1 expression in different BC subtypes.(C)qRT-PCR revealed a markedly lower level of MBNL1-AS1 in BC.(D)The expression levels of MBNL1-AS1were detected in different TNM stages.(E)qRT-PCR analysis of MBNL1-AS1 pattern in different cell lines. (F) 
Kaplan-Meier analysis indicated a better RFS in patients with highMBNL1-AS1expression. ${ }^{*} \mathrm{P}<0.05,{ }^{*} \mathrm{P}<$ 0.01

A

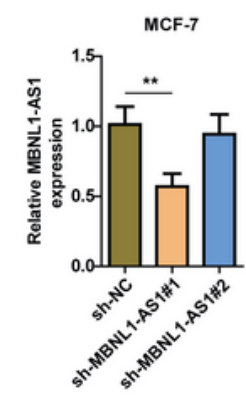

C
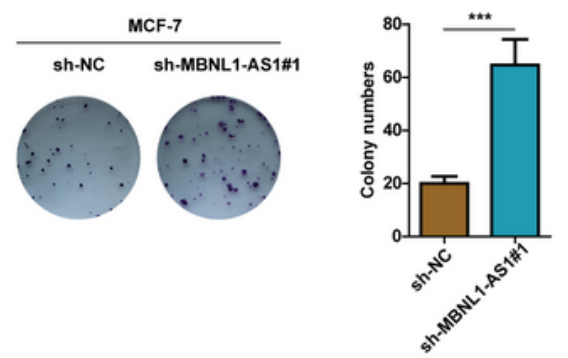

D
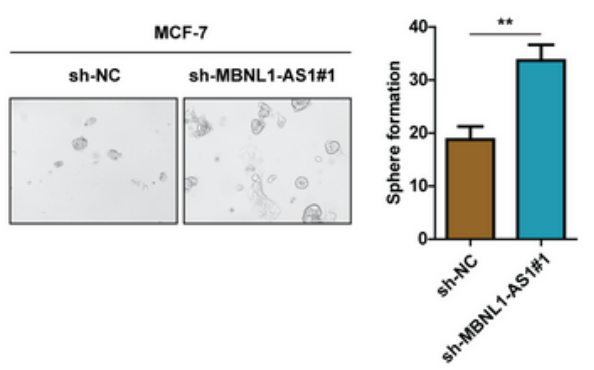

E
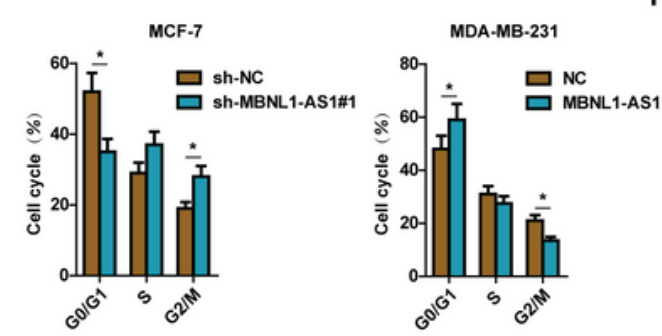

MCF-7

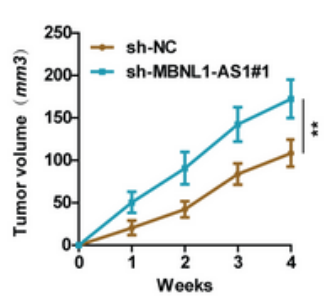

B
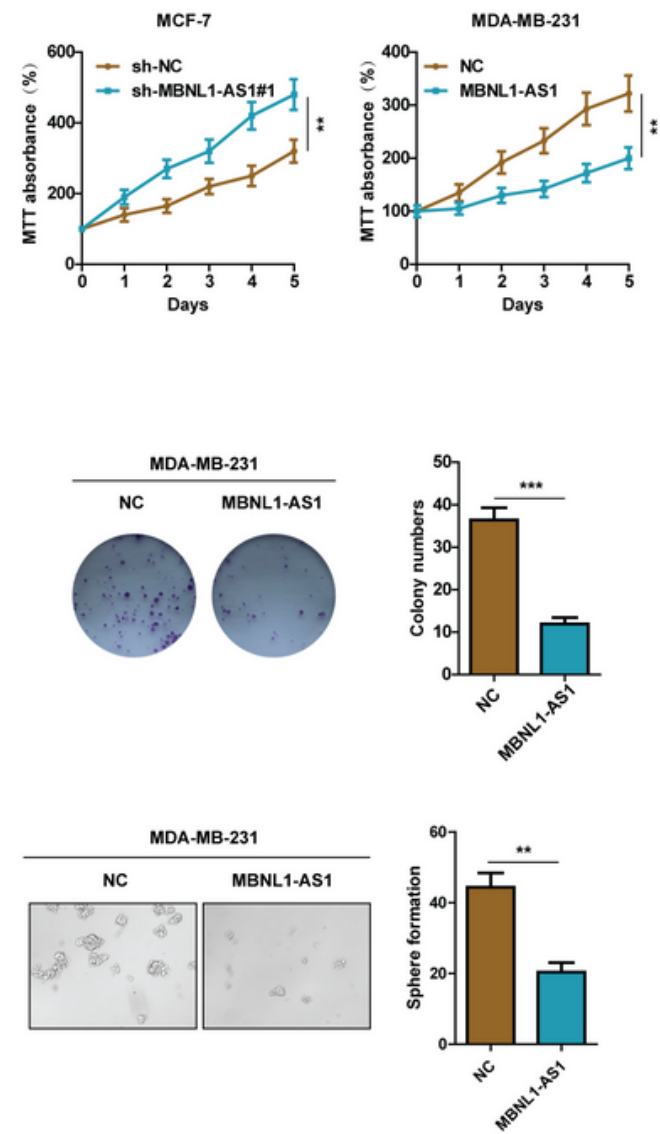

$\mathbf{F}$
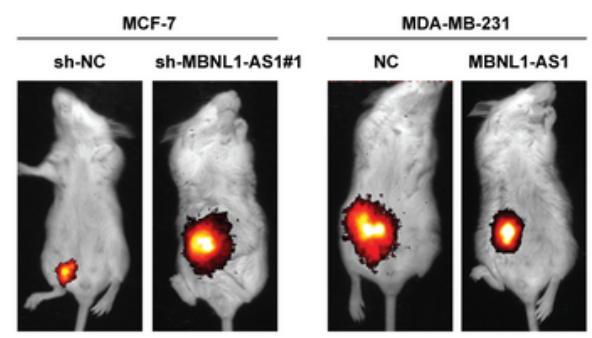

\section{Figure 2}

Biological functions of MBNL1-AS1 in BC.(A)qRT-PCR of MBNL1-AS1 in indicated cells transfected with shRNA-MBNL1-AS1\#1/\#2 (sh-MBNL1-AS1\#1/\#2) or shRNA-control (sh-NC) and MBNL1-AS1 or the 
scramble control sequence. MTT assays (B) and colony formation assays (C) were used to examine the proliferation of MCF-7 cells with MBNL1-AS1 knockdown and MDA-MB-231 cells with MBNL1-AS1 overexpression. (D) Number of tumor spheres inindicated cells. (E) The BC cells in different phases were detected by flow cytometry analysis. (F) Orthotopic breast tumor mice models were used to explore the growth of tumors. Tumor diameters were evaluated every week

A

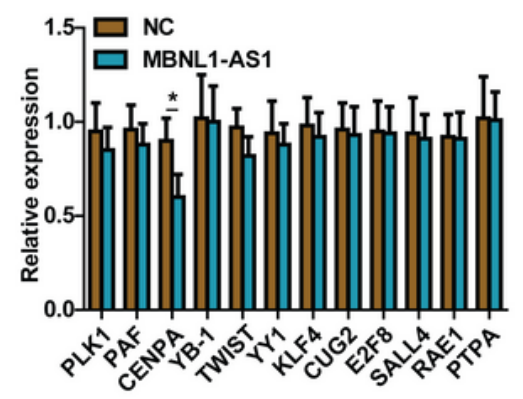

C

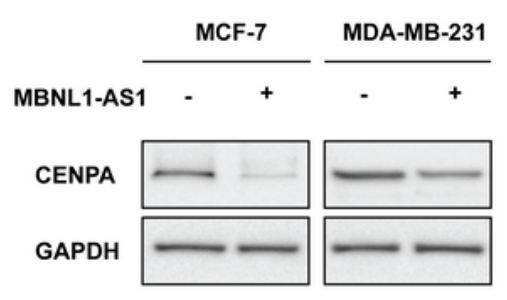

E
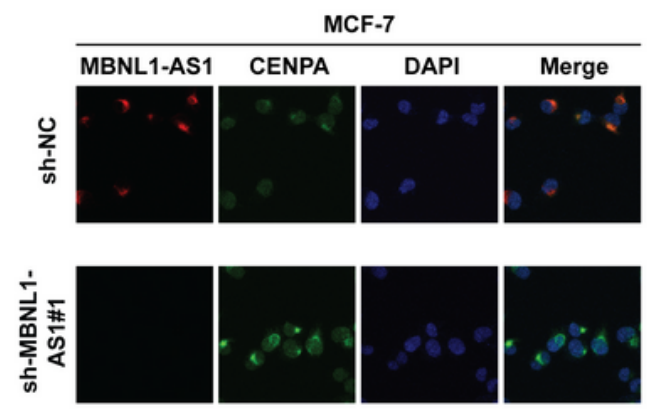

F

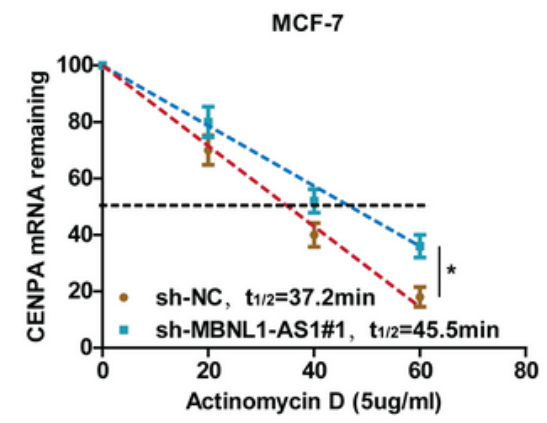

B

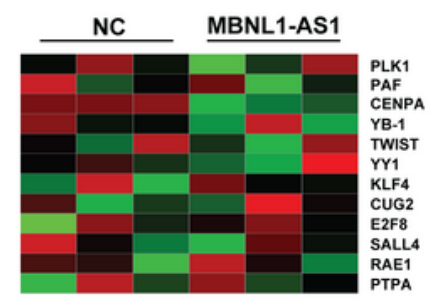

D

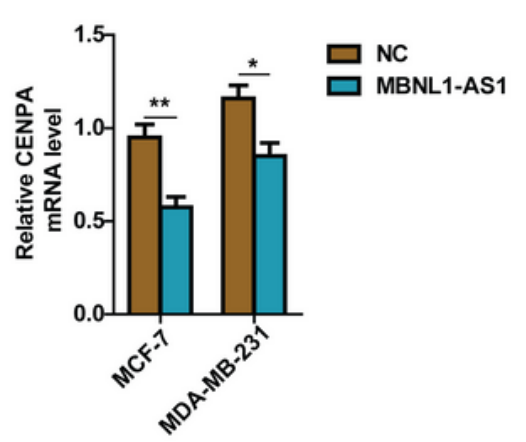

Figure 3 
MBNL1-AS1 inhibited the expression of CEPNA by reducing the stability of CENPA mRNA.The proliferation- and stemness-associated factors were detected and shown by Flexmapliquichip assays (A) and the heatmap (B).The expression levels of CENPA in the BC cells were tested by western blot assays (C) and qRT-PCR (D).(E)Subcellular localization in indicated cells was examined by combined IF/FISH assays. $(\mathrm{F})$ The abundance of CENPA in indicated cells treated with actinomycin D was detected by qRTPCR. * $\mathrm{P}<0.05, * * \mathrm{P}<0.01$

A

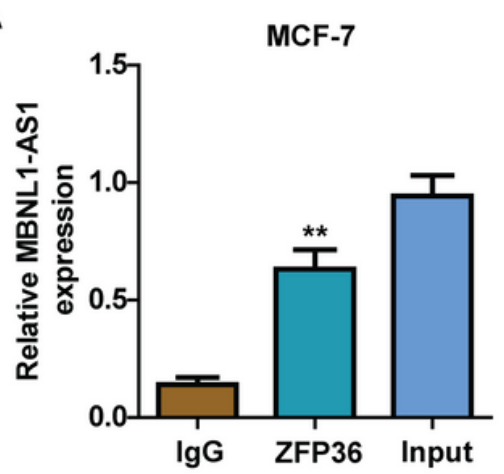

C

MCF-7
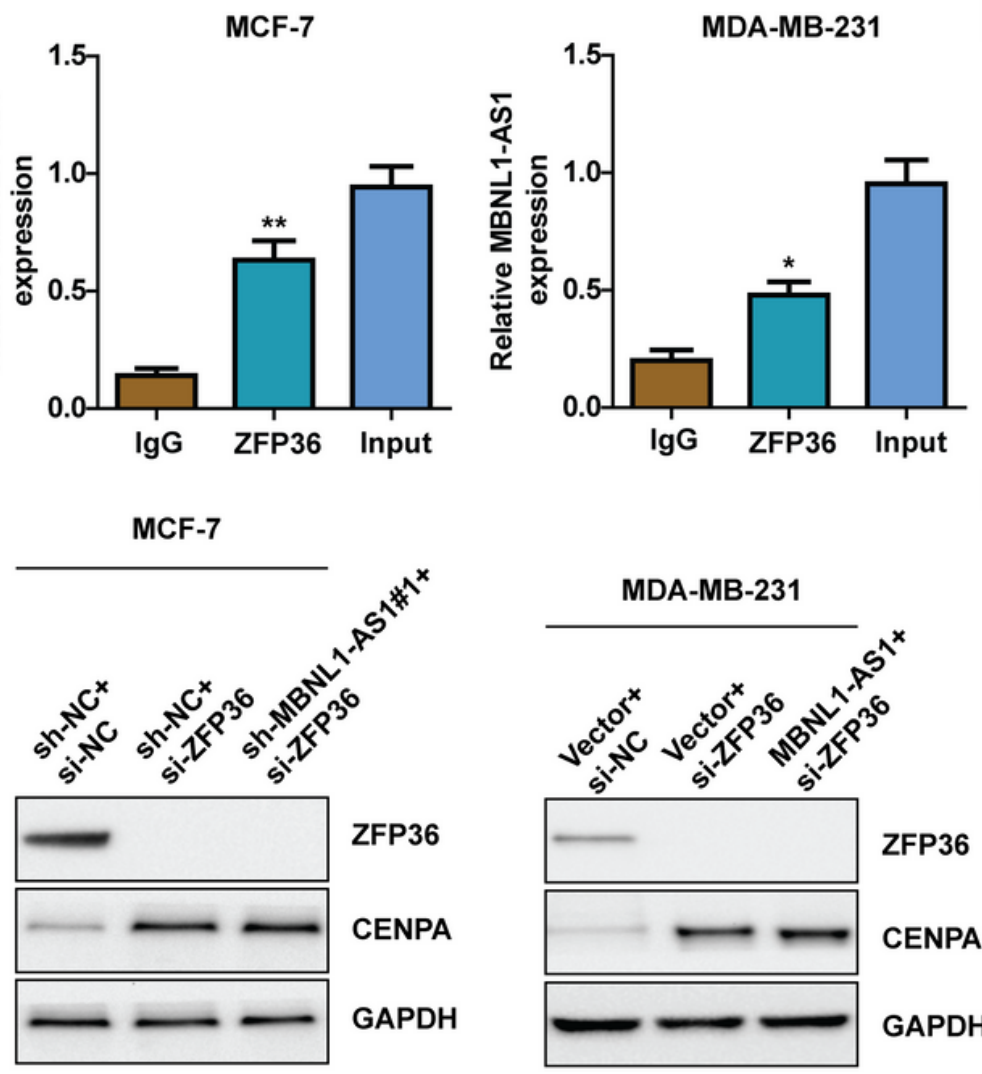

B

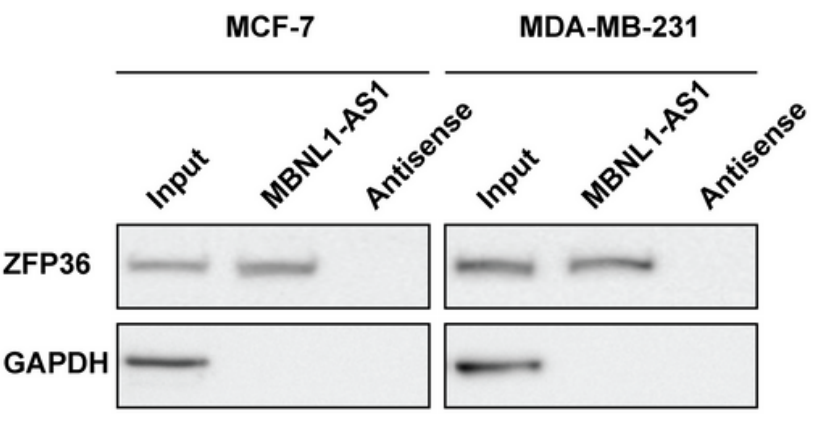

D

MDA-MB-231

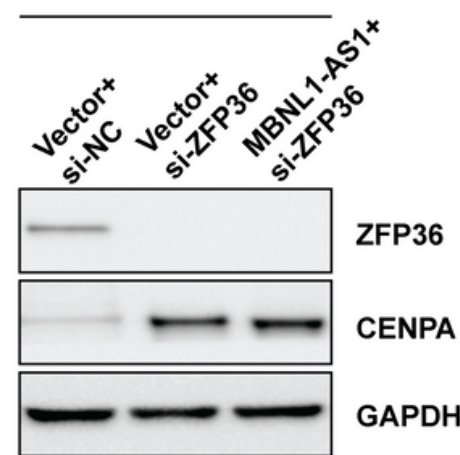

MCF-7

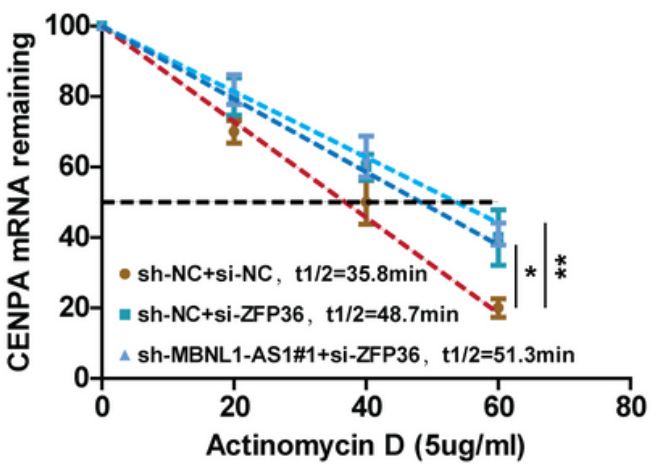

MDA-MB-231

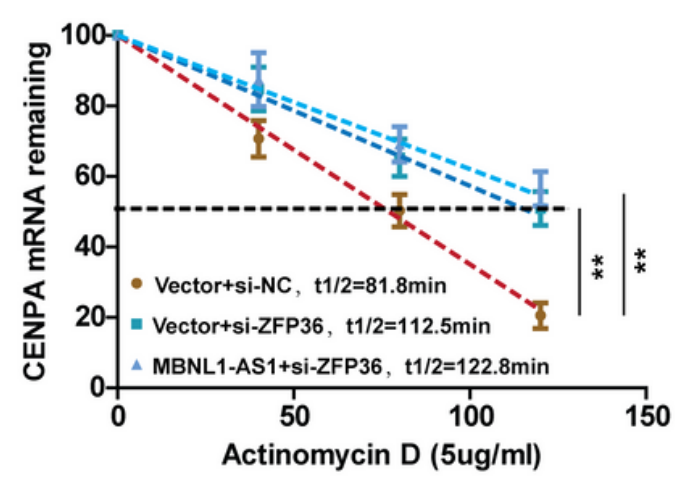

Figure 4 
MBNL1-AS1 interacted with ZFP36 and reduced the CENPA mRNA stability.(A) RIP assays and (B) RNA pull-down assays were used to examinethe combination of MBNL1-AS1 and ZFP36 in MCF-7 and MDAMB-231 cells. (C)The expression of ZFP36 and CENPAwere detected using western blot. (D)qRT-PCR assays were performed to examine the half-life of CENPA mRNA in the BC cells

A
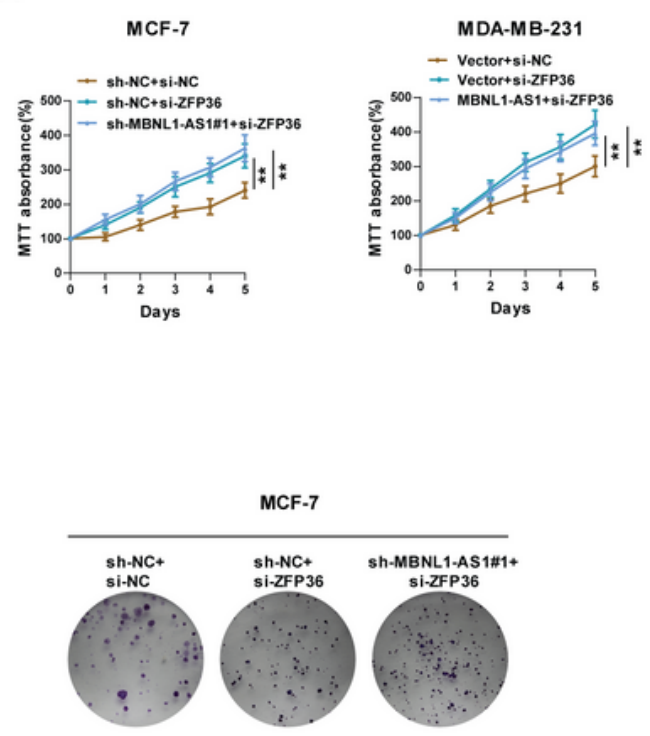

C
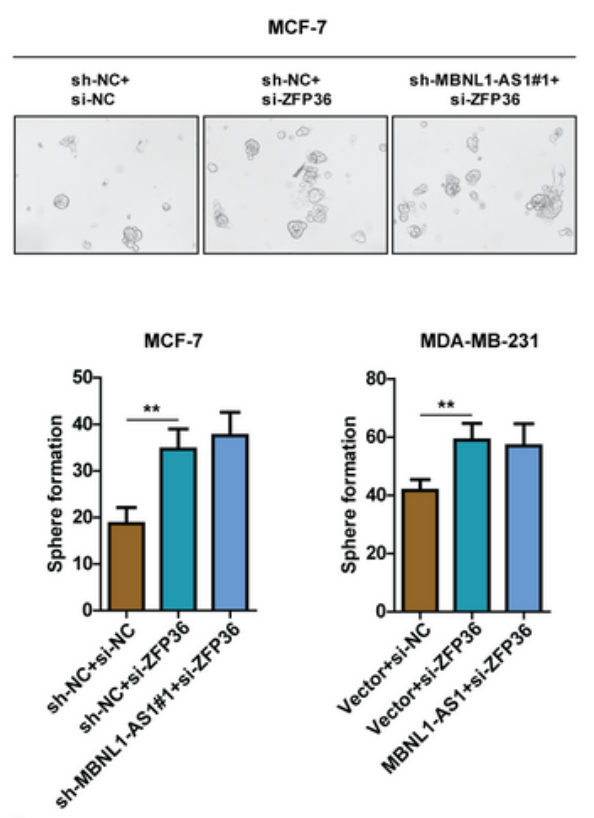

E

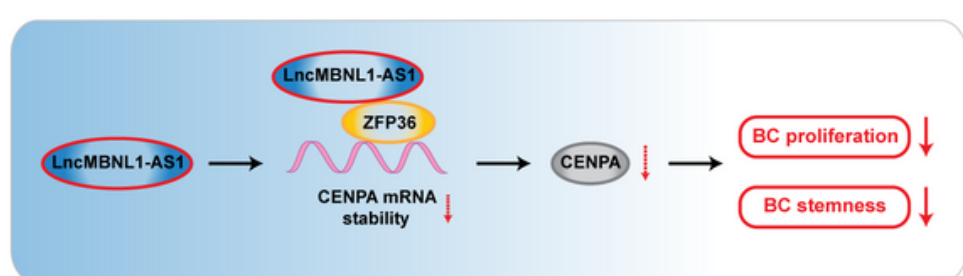

B
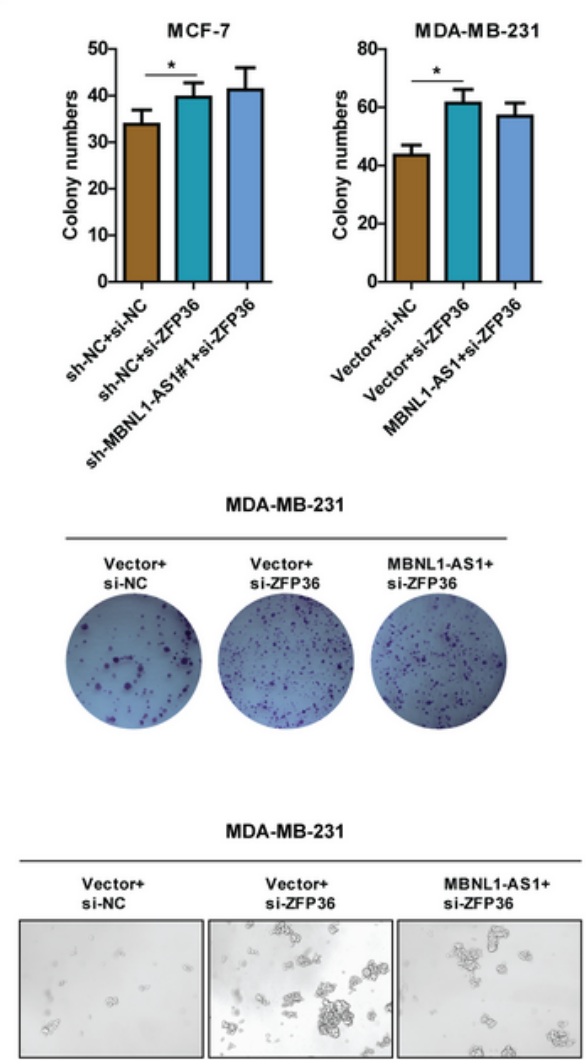

D

MCF-7

MDA-MB-231
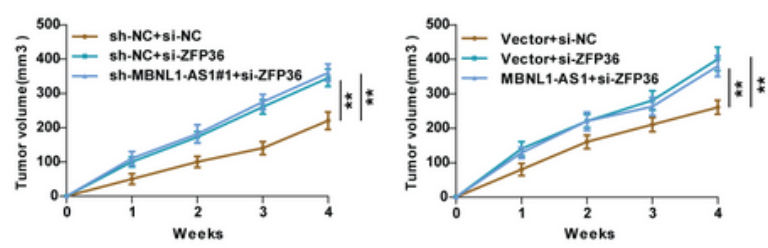

Figure 5 
MBNL1-AS1 suppressed proliferation and stemness abilities of BC cells by interacting with ZFP36.MTT assay (A) and colony formation assay (B) was performed to measure the proliferation of the cells. (C)Sphere formation assay wasperformed to analyze the stemness of indicated cells. (D) Orthotopic breast tumor mice models were used to explore the growth of tumors. Tumor diameters were evaluated every week.(E)The MBNL1-AS1/ZFP36/CENPA axis regulated proliferation and stemness in BC cells and then affected $B C$ progression 